\title{
On extremal self-dual codes of length 120
}

\author{
Javier de la Cruz*
}

\begin{abstract}
We prove that the only primes which may divide the order of the automorphism group of a putative binary self-dual doubly-even $[120,60,24]$ code are $2,3,5,7,19$, 23 and 29. Furthermore we prove that automorphisms of prime order $p \geq 5$ have a unique cycle structure.
\end{abstract}

\section{Introduction}

Throughout the paper all codes are assumed to be binary and linear, if not explicitly stated otherwise. Let $C=C^{\perp}$ be a self-dual code of length $n$ and minimum distance $d$. By results of Mallows-Sloane [13] and Rains [15], we have

$$
d \leq \begin{cases}4\left\lfloor\frac{n}{24}\right\rfloor+4 & \text { if } n \neq \equiv 22 \bmod 24 \\ 4\left\lfloor\frac{n}{24}\right\rfloor+6 & \text { if } n \equiv 22 \bmod 24\end{cases}
$$

and $C$ is called extremal if equality holds. The length $n$ of an extremal self-dual doublyeven code $C$ is bounded by $n \leq 3928$, due to a result of Zhang [20]. Furthermore, if in addition $n=24 m$, then $C$ is always doubly-even, as shown by Rains [15].

Already 1973 Sloane posed the question whether extremal self-dual codes of length 72 exist [17]. This is the first unsolved case if $24 \mid n$. Such codes are of particular interest since the supports of codewords of a given non-trivial weight form a 5-design according to the Assmus-Mattson Theorem [1]. Unfortunately, we know only two codes, the extended Golay code of length 24 and the extended quadratic residue code of length 48 . In order to find codes of larger length non-trivial automorphisms may be helpful. The following table shows what we know about the automorphism groups so far.

\begin{tabular}{c|c|c|l|l|} 
parameters & codes & $G$ & $\begin{array}{l}\text { (possible) primes } \\
\text { dividing }|G|\end{array}$ & reference \\
\hline$[24,12,8]$ & ext. Golay & $\mathrm{M}_{24}$ & $2,3,5,7,11,23$ & {$[12]$} \\
{$[48,24,12]$} & ext. QR & $\mathrm{PSL}(2,47)$ & $2,3,23,47$ & {$[9],[11]$} \\
{$[72,36,16]$} & $?$ & $|G| \leq 24$ & $2,3,5$ & {$[4],[8]$} \\
{$[96,48,20]$} & $?$ & $|G| \leq ?$ & $2,3,5$ & {$[7],[6]$}
\end{tabular}

* Javier de la Cruz is with the Universidad del Norte, Departamento de Matemáticas, Km 5 Vía Puerto Colombia, Barranquilla, Colombia (e-mail: jdelacruz@uninorte.edu.co). 
Looking at the table one is naturally attempted to ask.

Question 1 Suppose that a self-dual $[120,60,24]$ code $C$ exist. Are 2,3 and 5 the only primes which may divide the order of the automorphism group of $C$ ?

If this turns out be true we have more evidence that for large $m$ the automorphism group of an extremal self-dual code of length $n=24 m$ may contain only automorphisms of very small prime orders. As a consequence such a code has almost no symmetries, i.e., it is more or less a pure combinatorial object and therefore probably hard to find if it exists.

In this paper we investigate primes $p$ which may occur in the order of the automorphism group of an extremal self-dual code of length 120. In Section III we prove that the only primes which may divide the order of the automorphism group of a putative binary selfdual doubly even $[120,60,24]$ code $C$ are 2, 3, 5, 7, 19, 23 and 29. Moreover, we exclude some cycle types of automorphisms of order 3,5 and 7, which in particular shows that automorphisms of prime order $p \geq 5$ have a unique cycle structure. For involutions the possible cycle types are known by [2]. Thus, as the main result we obtain

Theorem 2 Let $C$ be an extremal self-dual code of length 120.

a) The only primes with may divide the order of the automorphism group of $C$ are $2,3,5,7,19,23$ and 29.

b) If $\sigma$ is an automorphism of $C$ of prime order $p$ then $p=2,3,5,7,19,23$ or 29 and its cycle structure is given by

\begin{tabular}{c|c|c}
$p$ & $\begin{array}{c}\text { number of } \\
p \text {-cycles }\end{array}$ & $\begin{array}{c}\text { number of } \\
\text { fixed points }\end{array}$ \\
\hline 2 & 48,60 & 24,0 \\
3 & $32,34,36,38,40$ & $24,18,12,6,0$ \\
5 & 24 & 0 \\
7 & 17 & 1 \\
19 & 6 & 6 \\
23 & 5 & 5 \\
29 & 4 & 4
\end{tabular}

In a forthcoming paper we will prove that automorphisms of order 3 act fixed point freely. Thus apart from (possibly) involutions all elements in $\operatorname{Aut}(C)$ of prime order have a unique cycle structure.

Theorem 2 is part of my PhD thesis [6]. 


\section{Preliminaries}

Let $C$ be a binary code with an automorphism $\sigma$ of odd prime order $p$. If $\sigma$ has $c$ cycles of length $p$ and $f$ fixed points, we say that $\sigma$ is of type $p-(c ; f)$. Without loss of generality we may assume that

$$
\sigma=(1,2, \ldots, p)(p+1, p+2, \ldots, 2 p) \ldots((c-1) p+1,(c-1) p+2, \ldots, c p) .
$$

Let $\Omega_{1}, \Omega_{2}, \ldots, \Omega_{c}$ be the cycle sets and let $\Omega_{c+1}, \Omega_{c+2}, \ldots, \Omega_{c+f}$ be the fixed points of $\sigma$. We put $F_{\sigma}(C)=\{v \in C \mid v \sigma=v\}$ and $E_{\sigma}(C)=\left\{v \in C \mid \operatorname{wt}\left(\left.v\right|_{\Omega_{i}}\right) \equiv 0 \bmod 2, i=\right.$ $1, \ldots, c+f\}$, where $\left.v\right|_{\Omega_{i}}$ is the restriction of $v$ on $\Omega_{i}$. With this notation we have

Lemma $3\left([9]\right.$, Lemma 2) $C=F_{\sigma}(C) \oplus E_{\sigma}(C)$.

There is an obvious relation between the weight distribution of $C$ and its subcode $F_{\sigma}(C)$, namely

Lemma 4 If $A_{i}$ and $A_{i}^{\prime}$ denotes the number of codewords of weight $i$ in $C$ resp. $F_{\sigma}(C)$ then $A_{i} \equiv A_{i}^{\prime} \bmod p$.

Proof: If $c \in C$ and $c \notin F_{\sigma}(C)$ then the size of the orbit of $c$ under $\sigma$ is divisible by $p$.

Clearly, a generator matrix of $C$ can be written in the form

$$
\operatorname{gen}(C)=\left(\begin{array}{cc}
X & Y \\
Z & 0
\end{array}\right) \quad \begin{aligned}
& \} \operatorname{gen}\left(F_{\sigma}(C)\right) \\
& \operatorname{gen}\left(E_{\sigma}(C)\right)
\end{aligned}
$$

where the first part of the matrix correspond to all coordinates which are moved by $\sigma$ and the second to the $f$ fixed points.

If $\pi: F_{\sigma}(C) \rightarrow \mathbb{F}_{2}^{c+f}$ denotes the map defined by $\pi\left(\left.v\right|_{\Omega_{i}}\right)=v_{j}$ for some $j \in \Omega_{i}$ and $i=1,2, \ldots, c+f$, then $\pi\left(F_{\sigma}(C)\right)$ is a binary $\left[c+f, \frac{c+f}{2}\right]$ self-dual code ([9], Lemma 1$)$.

Note that every binary vector of length $p$ can be identified with a unique polynomial in the factor algebra $\mathbb{F}_{2}[x] /\left(x^{p}-1\right)$ by $\left(v_{0}, v_{1}, \ldots, v_{p-1}\right) \mapsto v_{0}+v_{1} x+\ldots+v_{p-1} x^{p-1} \in \mathbb{F}_{2}[x]$. Furthermore, recall that the vector space of even-weight polynomials in $\mathbb{F}_{2}[x] /\left(x^{p}-1\right)$, which we denote by $P$, is a binary cyclic code of length $p$ generated by $x-1$. Let $E_{\sigma}(C)^{*}$ be the subcode of $E_{\sigma}(C)$ where the last $f$ coordinates have been deleted. For $v \in E_{\sigma}(C)^{*}$ we may consider the $p$-cycle

$$
v \mid \Omega_{i}=\left(v_{0}, v_{1}, \ldots, v_{p-1}\right) \quad(i=1, \ldots, c)
$$

as the polynomial

$$
\varphi\left(v \mid \Omega_{i}\right)(x)=v_{0}+v_{1} x+\ldots+v_{p-1} x^{p-1}
$$

in $P$. In this way we obtain a map $\varphi: E_{\sigma}(C)^{*} \rightarrow P^{c}$. Clearly, $\varphi\left(E_{\sigma}(C)^{*}\right)$ is a submodule of the $P$-module $P^{c}$. If the multiplicative order of 2 modulo $p$, usually denoted by $s(p)$, is $p-1$, then the check polynomial $1+x+x^{2}+\ldots+x^{p-1}$ of $P$ is irreducible over $\mathbb{F}_{2}$. Hence $P$ is an extension field of $\mathbb{F}_{2}$ with identity $e(x)=x+\ldots+x^{p-1}$ and $\varphi\left(E_{\sigma}(C)^{*}\right)$ is a code over the field $P$. 
Lemma 5 ([18, Theorem 3) Assume that $s(p)=p-1$. Then a binary code $C$ with an automorphism $\sigma$ of odd prime order $p$ is self-dual if and only if the following two conditions hold.

a) $\pi\left(F_{\sigma}(C)\right)$ is a binary self-dual code of length $c+f$.

b) $\varphi\left(E_{\sigma}(C)^{*}\right)$ is a self-dual code of length $c$ over the field $P$ under the inner product $u \cdot v=\sum_{i=1}^{c} u_{i} v_{i}^{q}$ for $q=2^{\frac{p-1}{2}}$.

Lemma 6 ([19], Theorem 3) Let $C$ be a binary self-dual code and let $\sigma$ be an automorphism of $C$ of odd prime order $p$. Then any Code, which can be obtained from $C$ by

(i) a substitution $x \rightarrow x^{t}$ in $\varphi\left(E_{\sigma}(C)^{*}\right)$, where $t$ is an integer with $1 \leq t \leq p-1$ or

(ii) a multiplication of the $j$ th coordinate of $\varphi\left(E_{\sigma}(C)^{*}\right)$ by $x^{t_{j}}$, where $t_{j}$ is an integer with $0 \leq t_{j} \leq p-1$ and $j=1,2, \ldots, c$,

is equivalent to $C$.

Lemma 5 and Lemma 6 are crucial to exclude the prime 59 in the order of the automorphism group of an extremal self-dual code of length 120. Most of the other primes can be excluded by the following two results.

Lemma 7 ([3], Theorem 7) If $C$ is a binary extremal self-dual code of length $24 m+2 r$ where $0 \leq r \leq 11, m \geq 2$, and $\sigma$ is an automorphism of $C$ of type $p$ - $(c ; f)$ for some prime $p \geq 5$ then $c \geq f$.

Lemma 8 ([19], Theorem 4) Let $C$ be a binary self-dual $[n, k, d]$ code and let $\sigma \in \operatorname{Aut}(C)$ be of type $p-(c ; f)$ where $p$ is an odd prime. If $g(s)=\sum_{i=0}^{s-1}\left\lceil\frac{d}{2^{i}}\right\rceil$ then

a) $p c \geq g\left(\frac{p-1}{2} c\right)$ and

b) $f \geq g\left(\frac{f-c}{2}\right)$ for $f>c$.

Lemma 9 ([3], Lemma 4) Let $C$ be a binary self-dual code of length $n$ and let $\sigma$ be an automorphism of $C$ of type $p-(c ; f)$ where $p$ is an odd prime. If $s(p)$ is even, then $c$ is even.

Let $C_{\pi_{1}}$ be the subcode of $\pi\left(F_{\sigma}(C)\right)$ which consists of all codewords which have support in the first $c$ coordinates and let $C_{\pi_{2}}$ be the subcode of $\pi\left(F_{\sigma}(C)\right)$ of all codewords which have support in the last $f$ coordinates. Then $\pi\left(F_{\sigma}(C)\right)$ has a generator matrix of the form

$$
\operatorname{gen}\left(\pi\left(F_{\sigma}(C)\right)\right)=\left(\begin{array}{cc}
A & O \\
O & B \\
D & E
\end{array}\right)
$$

where $(A O)$ is a generator matrix of $C_{\pi_{1}}$ and $(O B)$ is a generator matrix of $C_{\pi_{2}}, O$ being the appropriate size zero matrix. With this notation we have 
Lemma 10 ([10], Theorem 9.4.1) If $k_{1}=\operatorname{dim} C_{\pi_{1}}$ and $k_{2}=\operatorname{dim} C_{\pi_{2}}$, then the following holds true.

a) (Balance Principle) $k_{1}-\frac{c}{2}=k_{2}-\frac{f}{2}$.

b) $\operatorname{rank} D=\operatorname{rank} E=\frac{c+f}{2}-k_{1}-k_{2}$.

c) Let $\mathcal{A}$ be the code of length c generate by $A, \mathcal{A}_{D}$ the code of length c generated by the rows of $A$ and $D, \mathcal{B}$ the code of length $f$ generated by $B$, and $\mathcal{B}_{E}$ the code of length $f$ generated by the rows of $B$ and $E$. Then $\mathcal{A}^{\perp}=\mathcal{A}_{D}$ and $\mathcal{B}^{\perp}=\mathcal{B}_{E}$.

Lemma 11 Let $C$ be a binary self-dual code with minimum distance $d$ and let $\sigma \in \operatorname{Aut}(C)$ be of type $p-(c ; f)$ with $c=f<d$. Then $\pi\left(F_{\sigma}(C)\right)$ has a generator matrix of the form $\left(I \mid E^{\prime}\right)$ where $I$ is the identity matrix of size $c$.

Proof: We may write gen $\left(\pi\left(F_{\sigma}(C)\right)\right)$ as in (2). The condition $f<d$ implies that $B=0$. Since $c=f$, by the Balance Principle we see that $A=0$. Thus $D$ is regular and

$$
D^{-1} \operatorname{gen}\left(\pi\left(F_{\sigma}(C)\right)\right)=\left(I \mid E^{\prime}\right)
$$

is a generator matrix of $\pi\left(F_{\sigma}(C)\right)$.

Lemma 12 If $p$ is an odd prime and $C$ is a binary self-dual code with minimum distance $d$, then $\operatorname{Aut}(C)$ does not contain an automorphism $\sigma$ of type $p-(c ; f)$ with $c=f$ and $p+c<d$.

Proof: The condition $p+c<d$ implies $c=f<d$ and by Lemma11, we obtain a generator matrix of the form

$$
\operatorname{gen}\left(\pi\left(F_{\sigma}(C)\right)\right)=\left(I \mid E^{\prime}\right) .
$$

Let $v$ be any row vector of $\left(I \mid E^{\prime}\right)$. Then $\pi^{-1}(v)=c \in C$ has weight

$$
\mathrm{wt}(c) \leq p+f=p+c<d,
$$

a contradiction.

\section{Excluding primes in the automorphism group of extremal self-dual codes of length 120}

In this section $C$ always denotes a binary self-dual code with parameters [120,60,24]. The weight enumerator of the code $C$ is determined in [13] as

$$
W_{C}(y)=1+39703755 y^{24}+6101289120 y^{28}+475644139425 y^{32}+\ldots
$$

Suppose that there is a $\sigma \in \operatorname{Aut}(C)$ of prime order $p \geq 3$. According to Lemma 7, 8 and 9. the only possibilities for the type of $\sigma$ are the following. 


\begin{tabular}{c|c|c}
$\mathrm{p}$ & $\mathrm{c}$ & $\mathrm{f}$ \\
\hline 3 & $30,32,34,36$, & $30,24,18,12$, \\
& 38,40 & 6,0 \\
5 & $20,22,24$ & $20,10,0$ \\
7 & $15,16,17$ & $15,8,1$ \\
11 & 10 & 10 \\
13 & 9 & 3 \\
17 & 7 & 1 \\
19 & 6 & 6 \\
23 & 5 & 5 \\
29 & 4 & 4 \\
59 & 2 & 2
\end{tabular}

Lemma 13 The primes $p=11,13$ and $p=17$ do not divide $|\operatorname{Aut}(C)|$.

Proof: If $p=11$, then $c=f=10$ and $p+c=11+10=21<d=24$. Now we apply Lemma 12 to exclude $p=11$.

For $p=13$ we have $s(p)=12$. Thus, by Lemma 9, $c$ must be even, which contradicts $c=9$. Hence $p=13$ is not possible.

Note that $s(17)$ is even. Applying again Lemma 9 we obtain $c$ even, which contradicts $c=1$. Thus $p=17$ does not occur either.

Lemma 14 The prime 59 does not divide $|\operatorname{Aut}(C)|$.

Proof: Suppose that $\sigma \in \operatorname{Aut}(C)$ is of order 59. Thus $\sigma$ is of type 59- $(2 ; 2)$. We determine all possible generator matrices for $C$ with respect to the decomposition given in Lemma 3 and check by computer that the minimum distance is smaller than 24 in each case.

Step 1: Construction of all possible gen $(C)$.

By Lemma 5, the code $\pi\left(F_{\sigma}(C)\right)$ is self-dual and has parameters [4,2,2]. Thus

$$
\operatorname{gen}\left(\pi\left(F_{\sigma}(C)\right)\right)=\left(\begin{array}{ll|ll}
1 & 0 & 1 & 0 \\
0 & 1 & 0 & 1
\end{array}\right) .
$$

Consequently,

$$
\operatorname{gen}\left(F_{\sigma}(C)\right)=\left(\begin{array}{ll|ll}
\mathbf{1} & \mathbf{0} & 1 & 0 \\
\mathbf{0} & \mathbf{1} & 0 & 1
\end{array}\right)
$$

where $\mathbf{1}$ is the all-one vector and $\mathbf{0}$ the zero-vector of length 59 .

Next we determine gen $\left(E_{\sigma}(C)\right)$. Note that $s(59)=58$. Thus, by Lemma 5, the vector space $\varphi\left(E_{\sigma}(C)^{*}\right)$ is a self-dual $[2,1]$ code over the field $P=\mathbb{F}_{2^{58}}$ under the inner product $u \cdot v=u_{1} v_{1}^{29}+u_{2} v_{2}^{29}$. W.l.o.g. it is generated by some vector $(e(x), b(x)) \in P^{2}$ where $e(x)$ denotes the identity in $P$ and

$$
e(x)+b(x)^{2^{29}+1}=0 .
$$


Let $\alpha(x)$ be a generator of the multiplicative group of $P$. Writing $b(x)=\alpha(x)^{r}$ with $0 \leq r \leq 2^{58}-2$ we obtain

$$
\alpha(x)^{r\left(2^{29}+1\right)}=e(x) .
$$

Thus $r=\left(2^{29}-1\right) k$ for some $k \in \mathbb{N}_{0}$ and therefore

$$
b(x)=\alpha(x)^{r}=\left(\alpha(x)^{2^{29}-1}\right)^{k}=\delta(x)^{k}
$$

where $\delta(x)=\alpha(x)^{2^{29}-1}$ and $0 \leq k \leq 2^{29}$. It follows, by Lemma 3, that $C$ has a generator matrix of the form

$$
\operatorname{gen}(C)=\left(\begin{array}{cc|cc}
\mathbf{1} & \mathbf{0} & 1 & 0 \\
\mathbf{0} & \mathbf{1} & 0 & 1 \\
\hline[e(x)] & {\left[\delta(x)^{k}\right]} & 0 & 0
\end{array}\right)
$$

where $[e(x)]$ and $\left[\delta(x)^{k}\right]$ are circulant $58 \times 59$-matrices and $0 \leq k \leq 2^{29}$. We would like to mention here that some of the $2^{29}+1$ generator matrices may define equivalent codes.

Step 2: Reduction of the number of generator matrices gen $(C)$ in step 1.

Observe that $\langle\delta(x)\rangle$ is a subgroup of $P \backslash\{0\}$ of order $2^{29}+1=3 \cdot 59 \cdot 3033169$ which contains the subgroup $\langle x e(x)\rangle$ of order 59. Since the factor group $\langle\delta(x)\rangle /\langle x e(x)\rangle$ is generated by $\langle x e(x)\rangle \delta(x)$ we obtain

$$
\langle\delta(x)\rangle=\cup_{i=1}^{3 \cdot 3033169}\langle x e(x)\rangle \delta(x)^{i} .
$$

If we multiply $\delta(x)^{k}$ in step 1 by $x^{t}$ for some $0 \leq t \leq 58$, the corresponding generator matrix defines an equivalent code by Lemma 6 , part (ii). Thus, in $(*)$, we only have to consider the polynomials

$$
\delta(x)^{k} \text { for } k=1, \ldots, 9099507 .
$$

Next we apply the substitution $x \rightarrow x^{2}$ in $\varphi\left(E_{\sigma}(C)^{*}\right)$ which also leads to an equivalent code by Lemma 6, part (i). Clearly, this substitution applied to the generator $\left(e(x), \delta(x)^{k}\right)$ yields

$$
\left(e\left(x^{2}\right), \delta\left(x^{2}\right)^{k}\right)=\left(e(x), \delta(x)^{2 k}\right) .
$$

Now we divide $\mathbb{Z}_{9099507}$ into a disjoint union of orbits

$$
\operatorname{orb}(i)=\left\{2^{n} i \bmod 9099507 \mid i \in \mathbb{N}_{0}\right\}
$$

Observe that for all $j \in \operatorname{orb}(i)$ the corresponding codes $C$ are equivalent. With Magma one easily checks that there are exactly 156889 orbits. This shows that we only have to consider generator matrices

$$
\operatorname{gen}(C)=\left(\begin{array}{cc|cc}
\mathbf{1} & \mathbf{0} & 1 & 0 \\
\mathbf{0} & \mathbf{1} & 0 & 1 \\
\hline[e(x)] & {\left[\alpha(x)^{t\left(2^{29}-1\right)}\right]} & 0 & 0
\end{array}\right)
$$

where $t$ runs through a set of representatives of the 156889 orbits. In each case we find with Magma a codeword of minimum distance smaller 24 which completes the proof.

So far we have proved part a) of the Theorem. 


\section{The cycle strucures}

In this section we prove part b).

Lemma 15 Let $C$ be a self-dual $[120,60,24]$ code. Then $C$ has no automorphism of type $p-(c ; c)$ for $p=3,5$ and 7 .

Proof: According to the list in (41) we have to show that $C$ does not have an automorphism of type $3-(30 ; 30), 5-(20 ; 20)$ or $7-(15 ; 15)$.

Claim 1: $C$ has no automorphism of type $3-(30 ; 30)$.

Let $\sigma \in \operatorname{Aut}(C)$ of type $3-(30 ; 30)$. Then $\pi\left(F_{\sigma}(C)\right)$ is a self-dual $\left[60,30, d_{\pi}\right]$ code. According to (11) of the introduction we have $d_{\pi} \leq 12$. Now we take a generator matrix for $\pi\left(F_{\sigma}(C)\right)$ in the form of (2). Since $c=f$, we get $k_{1}=k_{2}$, by the Balance Principle (see Lemma [10). Note that $\mathcal{B}$ is a doubly even $\left[30, k_{2}, d^{\prime}\right]$ code with $d^{\prime}=24$ or $d^{\prime}=28$. If $k_{2} \geq 2$, then obviously $\pi\left(F_{\sigma}(C)\right)$, and therefore $C$ contains a codeword of weight less or equal 12 , a contradiction. Thus $k_{1}=k_{2} \leq 1$.

If $k_{2}=0$, then gen $\left(\pi\left(F_{\sigma}(C)\right)\right)=\left(I_{30} \mid E\right)$. Let $\left(e_{i} \mid v_{i}\right)$ denote the $i$-th row of $\left(I_{30} \mid E\right)$. Since $\operatorname{wt}\left(\pi^{-1}\left(e_{i} \mid v_{i}\right)\right)=3+\operatorname{wt}\left(v_{i}\right) \geq 24$, we get $\operatorname{wt}\left(v_{i}\right)=21,25$ or 29 . If $\operatorname{wt}\left(v_{i}\right)=29$ and wt $\left(v_{j}\right)=29$, then

$$
S_{v_{i}, v_{j}}=\left|\operatorname{supp}\left(v_{i}\right) \cap \operatorname{supp}\left(v_{j}\right)\right| \geq 28
$$

and therefore $\operatorname{wt}\left(\pi^{-1}\left(e_{i}+e_{j} \mid v_{i}+v_{j}\right)\right)=6+\operatorname{wt}\left(v_{i}+v_{j}\right) \leq 8$, a contradiction. In all other cases we get similarly a contradiction unless $\operatorname{wt}\left(v_{i}\right)=21$ for all $i=1, \ldots, 30$.

If $x=\left(e_{i} \mid v_{i}\right)$ and $y=\left(e_{j} \mid v_{j}\right)$, then $S_{x, y}=S_{v_{i}, v_{j}} \geq 12$. In case $S_{v_{i}, v_{j}}>12$ we obtain $\operatorname{wt}\left(\pi^{-1}(x+y)\right) \leq 6+17=23$, a contradiction. Consequently $S_{v_{i}, v_{j}}=12$ for all $i \neq j \in\{1, \ldots, 30\}$. Hence two vectors $v_{i}, v_{j}$ do not have a coordinate simultaneously zero. This implies that the dimension of $\operatorname{gen}\left(\pi\left(F_{\sigma}(C)\right)\right)$ is at most 3 , a contradiction.

If $k_{2}=1$, then $\pi\left(F_{\sigma}(C)\right)$ has a generator matrix of the form

$$
\left(\begin{array}{cc}
a & 0 \ldots 0 \\
0 \ldots 0 & b \\
D & E
\end{array}\right)
$$

where $\operatorname{wt}(b)=24$ or 28 . Since $C$ is doubly even, $\operatorname{wt}(a) \in\{8,12,16,20,24,28\}$. Suppose that $\operatorname{wt}(a)=28$. Then $\operatorname{wt}\left(\pi^{-1}(a \mid b)\right) \geq 108$ which implies that $(a \mid b)$ is the all-one vector, a contradiction to $\operatorname{wt}(a)=28$. Thus $\operatorname{wt}(a) \leq 24$. Therefore $a$ contains in at least 6 positions 0 . Consequently, there are at least 6 vectors of the form $z_{i}=(0,0, \ldots, 1, \ldots, 0,0) \in \mathbb{F}_{2}^{30}$, which are orthogonal to $a$. By Lemma $10 \mathrm{c}$ ), we obtain $z_{i} \in \mathcal{A}^{\perp}=\mathcal{A}_{D}$. The contradiction now follows as in case $k_{2}=0$.

Claim 2: $C$ has no automorphism of type $5-(20 ; 20)$.

Note that $p=5 \equiv 1 \bmod 4$. Thus, by ([9], Lemma 1$)$, the space $\pi\left(F_{\sigma}(C)\right)$ is a doubly even self-dual $\left[40,20, d_{\pi}\right]$ code. Furthermore $c=f=20<d$. According to Lemma 11 we can take a generator matrix of $\pi\left(F_{\sigma}(C)\right)$ of the form $\operatorname{gen}\left(\pi\left(F_{\sigma}(C)\right)\right)=\left(I \mid E^{\prime}\right)$. If $x=\left(1,0,0, \ldots, 0 \mid e_{1}\right)$ and $y=\left(0,1,0, \ldots, 0 \mid e_{2}\right)$ denote the first respectively the second row of $\left(I \mid E^{\prime}\right)$, then

$$
\operatorname{wt}\left(\pi^{-1}(x)\right)=\operatorname{wt}\left(\pi^{-1}\left(\left(1,0,0, \ldots, 0 \mid e_{1}\right)\right)=5+\operatorname{wt}\left(e_{1}\right) \geq 24 .\right.
$$


Therefore $19 \leq \mathrm{wt}\left(e_{1}\right) \leq 20$. Since $C$ and $\pi\left(F_{\sigma}(C)\right)$ are doubly even we obtain $\mathrm{wt}\left(e_{1}\right)=19$. Similarly $\operatorname{wt}\left(e_{2}\right)=19$. This implies that $\operatorname{wt}\left(e_{1}+e_{2}\right) \leq 2$. Hence

$$
\operatorname{wt}\left(\pi^{-1}(x+y)\right)=\operatorname{wt}\left(\pi^{-1}\left(1,1,0, \ldots, 0 \mid e_{1}+e_{2}\right)\right)=2 \cdot 5+\operatorname{wt}\left(e_{1}+e_{2}\right) \leq 12,
$$

a contradiction.

Claim 3: $C$ has no automorphism of type $7-(15 ; 15)$.

Since $c=f=15$ and $p+c=7+15=22<d=24$ we may apply Lemma 12 to see that there is no automorphism of type $7-(15 ; 15)$.

Lemma 16 A self-dual $[120,60,24]$ code does not have an automorphism of type $5-(22 ; 10)$.

Proof: Suppose that $\sigma \in \operatorname{Aut}(C)$ is of type 5 - $(22 ; 10)$. Then $\pi\left(F_{\sigma}(C)\right)$ is a self-dual $\left[32,16, d_{\pi}\right]$ code. Furthermore, $\pi\left(F_{\sigma}(C)\right)$ is doubly even, by ([9], Lemma 1$)$, since $p \equiv$ $1 \bmod 4$. According to (11), we have $d_{\pi} \leq 8$. If we write $d_{\pi}=x+y$ where $x$ is the number of $1 \mathrm{~s}$ in the first $c=22$ coordinates of a minimal weight codeword and $y$ is the number of $1 \mathrm{~s}$ in the last $f=10$ coordinates, then $x+y \leq 8$ and $5 x+y \geq 24$. This forces $x \geq 4$ and $d_{\pi}=8$. Hence $\pi\left(F_{\sigma}(C)\right)$ is an extremal self-dual doubly even code of length 32 . By ([16], p. 262) there are (up to isometry) exactly five such codes, denoted by $C 81$ (extended quadratic residue code), $C 82$ (Reed-Muller code), $C 83, C 84$ and $C 85$. To see that no one of these codes can occur as $\pi\left(F_{\sigma}(C)\right)$ we proceed as follows.

Let $C_{0}$ denote one of these code. We do not know which coordinates belong to the fixed points of $\sigma$. We know only the number, namely 10. Therefore we choose all possible 10 -subsets of $1, \ldots, 32$ and take them as the coordinates of fixed points. In each case we construct $\pi^{-1}\left(C_{0}\right)$ and compute the minimum distance with MAGMA. In turns out that all distances are strictly less than 24 . Thus none of the five extremal doubly even codes of length 32 can occur as $\pi\left(F_{\sigma}(C)\right)$, a contradiction.

Lemma 17 C has no automorphism of type 7-(16;8).

Proof: Let $\sigma$ be an automorphism of type $7-(16 ; 8)$. Then $\pi\left(F_{\sigma}(C)\right)$ is a self-dual $\left[24,12, d_{\pi}\right]$ code. According to (1) we have $d_{\pi} \leq 8$. If $d_{\pi}=x+y$ where $x$ is again the number of $1 \mathrm{~s}$ in the left 16 coordinates and $y$ is the number of $1 \mathrm{~s}$ in the right 8 coordinates of a codeword of minimal weight, then $x+y \leq 8$ and $7 x+y \geq 24$. Therefore $x \geq 3$ and $d_{\pi}=4,6$ or 8 . In total there are 30 self-dual $\left[24,12, d_{\pi}\right]$ codes (see [14, [5]), one with $d_{\pi}=8$, one with $d_{\pi}=6$ and 28 with $d_{\pi}=4$.

If $d_{\pi}=8$ then $\pi\left(F_{\sigma}(C)\right)$ is the Golay code. The weight enumerator of the Golay code is $1+759 y^{8}+2576 y^{12}+759 y^{16}+y^{24}$. We know that a vector of $F_{\sigma}(C)$ of weight 28 can be formed only by vectors of $\pi\left(F_{\sigma}(C)\right)$ of weight 4 and 10 since $28=4 \cdot 7+0$ and $28=3 \cdot 7+7$. Therefore, $F_{\sigma}(C)$ has no codewords of weight 28. But this contradicts the fact that the number $A_{28}$ (see (3) ) of codewords of $C$ of weight 28 satisfies $A_{28}=6101289120 \equiv 3 \bmod 7$, by Lemma 4 .

If $d_{\pi}=6$ then $\pi\left(F_{\sigma}(C)\right)$ is the code $Z_{24}$ (see [5], TABLE E). In this case we take all 
possibilities for the 8 fixed points and construct $\pi^{-1}\left(Z_{24}\right)$. In all situations we find with MAGMA a vector of weight less than 24 or not divisible by 4 .

Thus we are left with the case $d_{\pi}=4$. Now observe the following fact. If a vector of $\pi\left(F_{\sigma}(C)\right)$ has weight 4 , then all non-zero coordinates correspond to cycles, since $C$ has minimum distance 24. So, if $\pi\left(F_{\sigma}(C)\right)$ has components $d_{n}$ or $e_{n}$ (for notation see [14]), then the corresponding coordinates are cycles. With this observation we easily see that $\sigma$ has less than 8 fixed points unless $\pi\left(F_{\sigma}(C)\right.$ is of type $X_{24}$ or $Y_{24}$. The case $\pi\left(F_{\sigma}(C)\right)=X_{24}$ can not occur since it yields a vector of weight $30 \mathrm{in} C$. The final case $Y_{24}$ has been excluded with MAGMA similar to the case $Z_{24}$.

Acknowledgment The author would like to thank Professor Willems for their contributions and valuable suggestions.

\section{References}

[1] E.F. Assmus, Jr. and H.F. Mattson Jr., New 5-designs, J. Combin. Theory 6 (1969), $122-151$.

[2] S. Bouyuklieva, On the automorphisms of order 2 with fixed points for the extremal self-dual codes of length 24m, Des. Codes and Crypt. 25 (2002), 5-13.

[3] S. Bouyuklieva, A. Malevich and W. Willems, Automorphisms of extremal self-dual codes, IEEE Trans. Inform. Theory 56 (2010), 2091-2096.

[4] E.A. O'Brien and W. Willems, On the automorphism group of a binary self-dual doubly-even [72,36,16] code, IEEE Trans. Inform. Theory 57 (2011), 4445-4451.

[5] J.H. Conway, V. Pless, and N. J. A. Sloane, The binary self-dual codes of length up to 32:A revised enumeration, J. Comb. Theory, Ser. A, vol. 60 1992, 183-195.

[6] J. de la Cruz, Über die Automorphismengruppe der extremale Codes der Längen 96 und 120. Ph.D.,Otto-von-Guericke Universität, Magdeburg, Germany, 2012.

[7] R. Dontcheva, On the doubly-even self-dual codes of length 96, IEEE Trans. Inform. Theory 48 (2002), 557-560.

[8] T. Feulner, G. Nebe, The automorphism group of an extremal [72,36,16] code does not contain $Z_{7}, Z_{3} \times Z_{3}$, or $D_{10}$. (preprint, arXiv:1110.6012v1)

[9] W.C. Huffman, Automorphisms of codes with application to extremal doubly-even codes of lenght 48, IEEE Trans. Inform. Theory 28 (1982), 511-521.

[10] W.C. Huffman and V. Pless, Fundamentals of Error-Correcting Codes, Cambridge University Press, Cambridge 2003.

[11] J.S. Leon, V. Pless and N.J.A. Sloane, Duadic codes, IEEE Trans. Inform. Theory 30 (1984), 709-714. 
[12] J. MacWilliams and N.J.A. Sloane, The Theory of Error-Correcting Codes, NorthHolland, Amsterdam 1977.

[13] C.L. Mallows and N.J.A. Sloane, An upper bound for self-dual codes, Inform. and Control 22 (1973), 188-200.

[14] V. Pless and N.J.A. Sloane, On the classification and enumeration of self-dual codes, J. Comb. Theory, Ser. A, 18 (1975), 313-335.

[15] E.M. Rains, Shadow bounds for self-dual-codes, IEEE Trans. Inform. Theory 44 (1998), 134-139.

[16] E.M. Rains and N.J.A. Sloane, Self-dual codes, in Handbook of coding theory, Volume 1, Elsevier 1998, 177-294.

[17] N.J.A. Sloane, Is there a $[72,36], d=16$ self-dual code? IEEE Trans. Inform. Theory, 19 (1973), 251.

[18] V.Y. Yorgov, Binary self-dual codes with automorphisms of odd order, Probl. Pered. Inform. 19 (1983), 11-24, Russian.

[19] V.Y. Yorgov, A method for constructing inequivalent self-dual codes with applications to length 56, IEEE Trans. Inform. Theory 33 (1987), 77-82.

[20] S. Zhang, On the nonexistence of extremal self-dual codes, Discrete Appl. Math. 91 (1999), 277-286. 